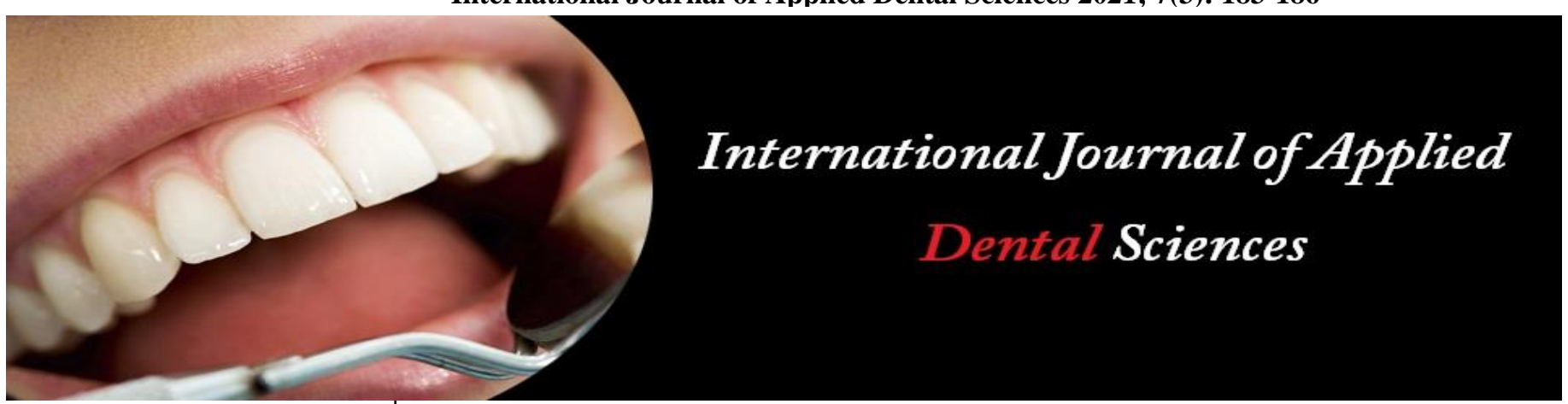

ISSN Print: 2394-7489

ISSN Online: 2394-7497

IJADS 2021; 7(3): 183-186

(C) 2021 IJADS

www.oraljournal.com

Received: 25-05-2021

Accepted: 27-06-2021

Shubham Kajani

Post Graduate Student,

Department of Conservative Dentistry and Endodontics,

Bapuji Dental College and

Hospital, Davangere,

Karnataka, India

Sophia Thakur

Professor, Department of

Conservative Dentistry and

Endodontics, Bapuji Dental

College and Hospital, Davangere,

Karnataka, India
Corresponding Author: Shubham Kajani Post Graduate Student,

Department of Conservative Dentistry and Endodontics,

Bapuji Dental College and

Hospital, Davangere,

Karnataka, India

\section{Biomimetic restoration of mandibular and maxillary molars using the stamp technique: A case series}

\section{Shubham Kajani and Sophia Thakur}

DOI: $\underline{\text { https://doi.org/10.22271/oral.2021.v7.i3c.1299 }}$

\section{Abstract}

A favourable occlusion is essential for better adaptation of the teeth to the surrounding neuromuscular and temporo-mandibular structures functionally. If this comes in support with an aesthetic rehabilitation of the teeth, it will enhance the normal functioning of the patient in all the aspects. The stamp technique is a viable, simple and accurate procedure for the restoration of class 1 carious lesion in an aesthetic and biomimetic manner, which minimizes any sort of post-procedural discomfort to the patient.

Keywords: occlusion, stamp, biomimetic

\section{Introduction}

With the advent of modern dentistry, and the universal demand for aesthetic restorations, there has been a shift from the traditional silver amalgam restoration to a more bio-mimetic toothcolored filling ${ }^{[1]}$.

Depending upon the loss of tooth structure, either a direct or an indirect restoration is advocated, wherein, a direct restoration poses several challenges such as obtaining proper contact and contour, achieving proper isolation, time consumption and technique sensitivity ${ }^{[2]}$. However, a considerable attention is not given to achieving a precise occlusion. Discrepancies in occlusion after a solution can prove detrimental to the treatment outcomes, and cause varied responses ranging from post-operative pain, difficulty in mastication, eventually fracturing the restoration to craniomandibular disturbances ${ }^{[3]}$.

In order to achieve proper occlusal harmony, and precise details in occlusal anatomy, a rather simple and a novel technique called the 'Stamp' technique has been introduced, to restore small to moderate caries in the posterior quadrant region. It involves the preparation of an index prior to caries excavation, and it is consequently submerged into the last increment of composite, before curing it ${ }^{[2,3]}$. Its principal advantage is to create an exact replica of the occlusal topography pre-operatively ${ }^{[4]}$.

This case series highlights the use of the 'Stamp' technique in the restoration of class I caries and its special consideration to "Biomimetics".

\section{Case Report 1}

A 30-year-old female patient reported to the Department of Conservative Dentistry and Endodontics with a chief complaint of decayed tooth in the lower left back tooth region. No pain was associated; however, a complaint of food lodgment was also noted. Following clinical and radiological examination, a class I caries involving the enamel and dentin, and a diagnosis of asymptomatic reversible pulpitis was made in relation to tooth 37 . The tooth was isolated using rubber dam and teflon tape. (Fig 1b) The stamp fabrication procedure was initiated by using gingival barrier, and syringing it into the grooves and fissures of the tooth. It was then cured, and, followed by placement of a microbrush, and using a little more gingival barrier, followed by curing it. (Fig 1c) The stamp, was then retrieved with the help of a blunt instrument. (Fig 1d) The cavity preparation was done, followed by etching and dentin bonding agent application. (Fig 1e,f,g) The cavity was then overfilled with composite (3M ESPE) restorative material. (Fig $1 \mathrm{~h}$ ) It was followed by placement of teflon tape and stamping the composite. 
(Fig 1i) The immediate post-stamping picture is shown. (Fig 1j) The restoration was completed with minimal finishing and polishing procedures, and minor occlusal adjustments. (Fig $1 \mathrm{k})$

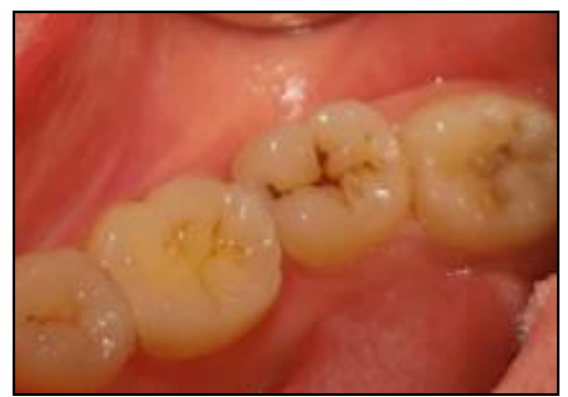

Fig 1a: Pre-operative photograph (without isolation)

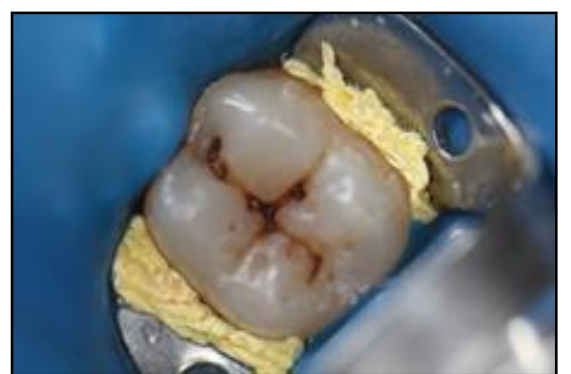

Fig 1b: Pre-operative photograph (With isolation)

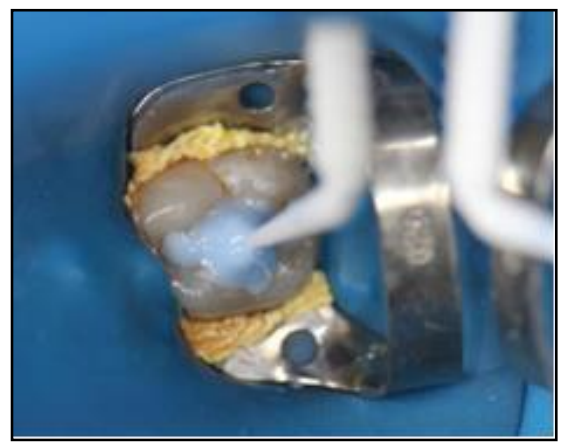

Fig 1c: Stamp placement using gingival barrier

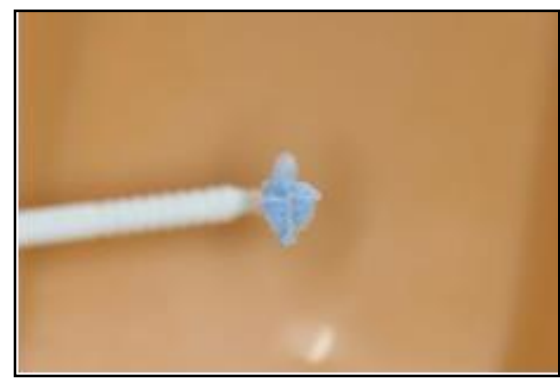

Fig 1d: Stamp retrieval

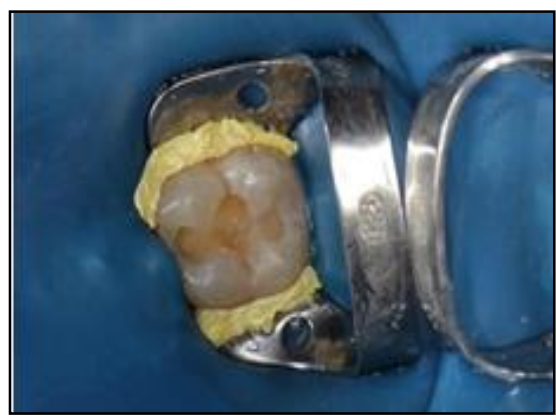

Fig 1e: Caries removal

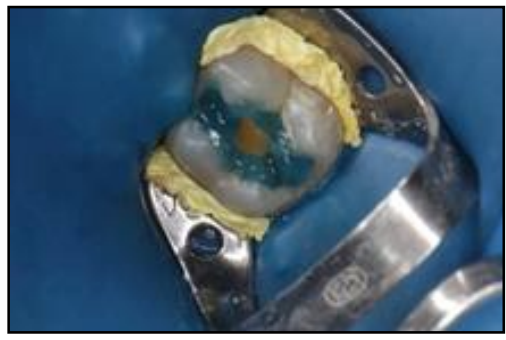

Fig 1f: Acid etching with $37 \%$ phosphoric acid

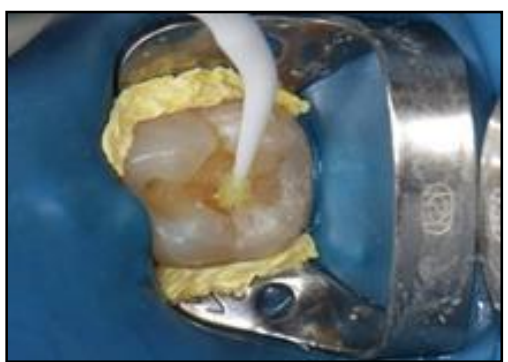

Fig 1g: Dentin bonding agent application

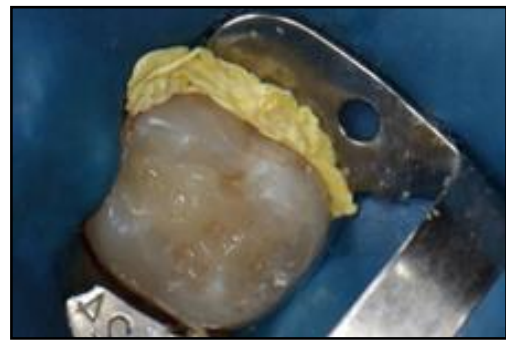

Fig 1h: Overfilling with composite

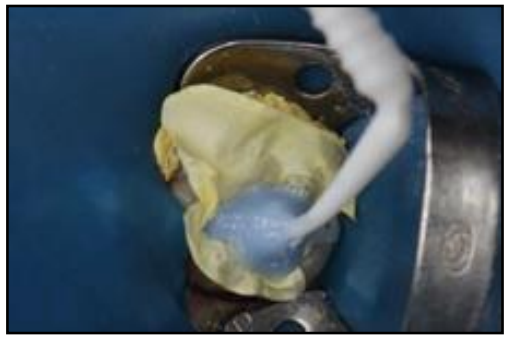

Fig 1i: Stamping the composite

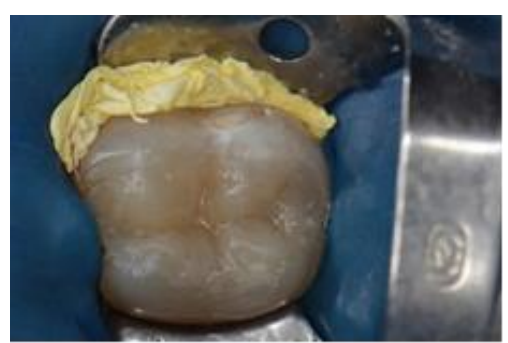

Fig 1j: Immediate post-operative photograph

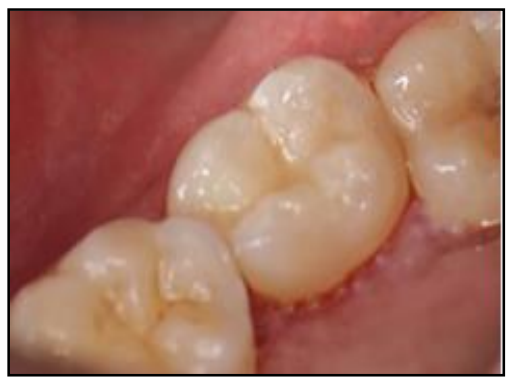

Fig 1k: After finishing and polishing 


\section{Case Report 2}

A 16-year-old female patient reported to the Department of Conservative Dentistry and Endodontics with a chief complaint of decayed tooth in the upper left back tooth region. No pain was associated, however, a complaint of food lodgment was also noted. Following clinical and radiological examination, a class I caries involving the enamel and dentin, and a diagnosis of asymptomatic reversible pulpitis was made in relation to tooth 27 . The tooth was isolated using rubber dam and teflon tape. The stamp fabrication procedure was initiated by using gingival barrier, and syringing it into the grooves and fissures of the tooth. It was then cured, and, followed by placement of a microbrush, and using a little more gingival barrier, followed by curing it. The stamp, was then retrieved with the help of a blunt instrument. The tooth was isolated using rubber dam and teflon tape. (Fig 2b) The stamp fabrication procedure was initiated by using gingival barrier, and syringing it into the grooves and fissures of the tooth. It was then cured, and, followed by placement of a microbrush, and using a little more gingival barrier, followed by curing it. (Fig 2c) The stamp, was then retrieved with the help of a blunt instrument. (Fig 2d) The cavity preparation was done, followed by etching and dentin bonding agent application. (Fig 2e,f,g) The cavity was then overfilled with composite (3M ESPE) restorative material. (Fig 2h) It was followed by placement of teflon tape and stamping the composite. (Fig 2i) The immediate post-stamping picture is shown. (Fig 2j) The restoration was completed with minimal finishing and polishing procedures, and minor occlusal adjustments. (Fig 1k)

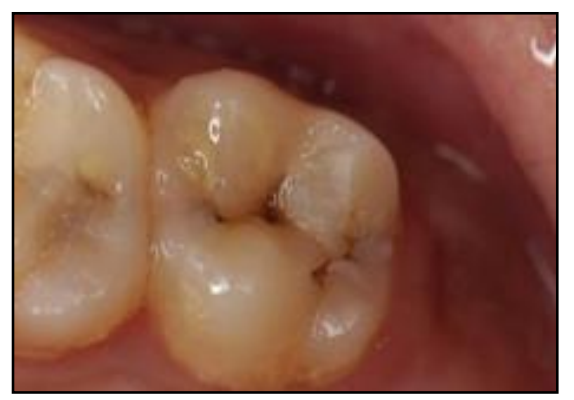

Fig 2a: Pre-operative photograph (without isolation)

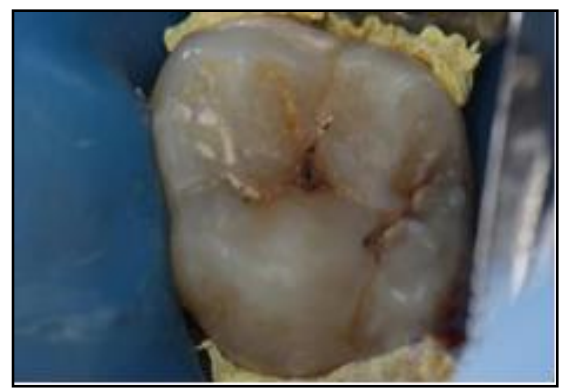

Fig 2b: Pre-operative photograph (With isolation)

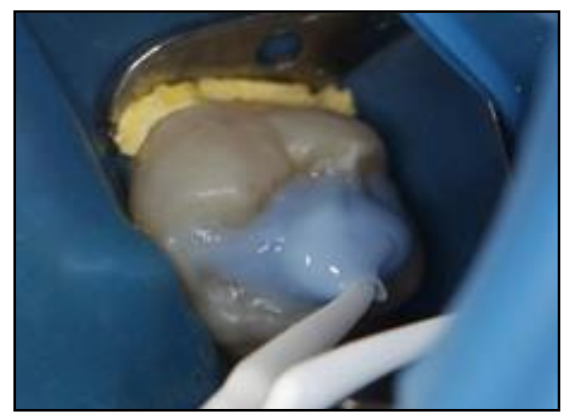

Fig 2c: Stamp placement using gingival barrier

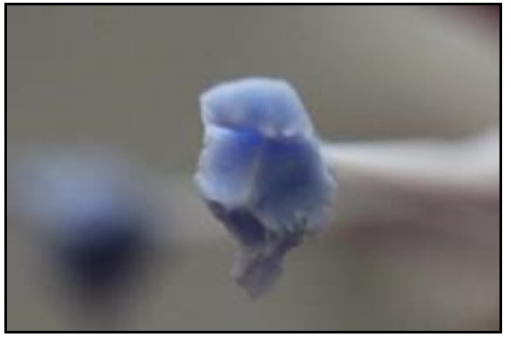

Fig 2d: Stamp retrieval

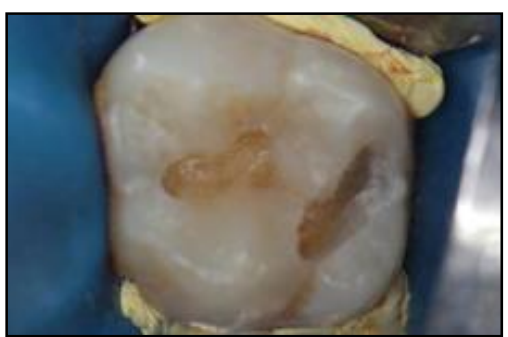

Fig 2e: Caries removal

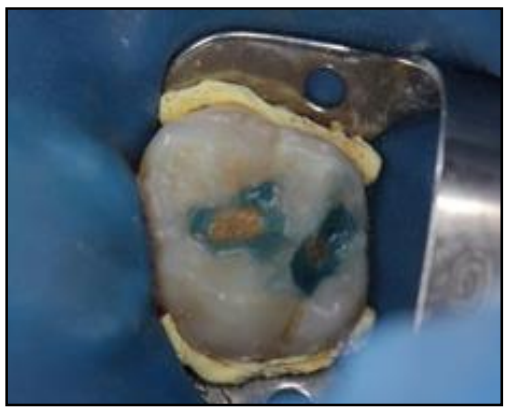

Fig 2f: Acid etching with $37 \%$ phosphoric acid

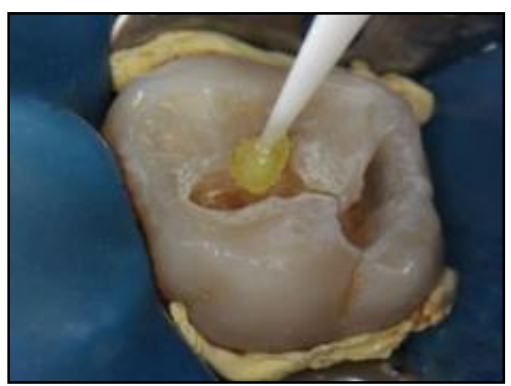

Fig 2g: Dentin bonding agent application

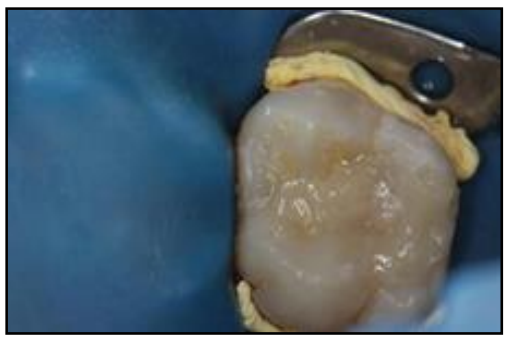

Fig 2h: Overfilling with composite

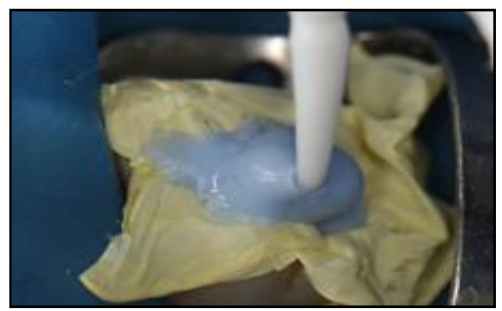

Fig 2i: Stamping the composite 


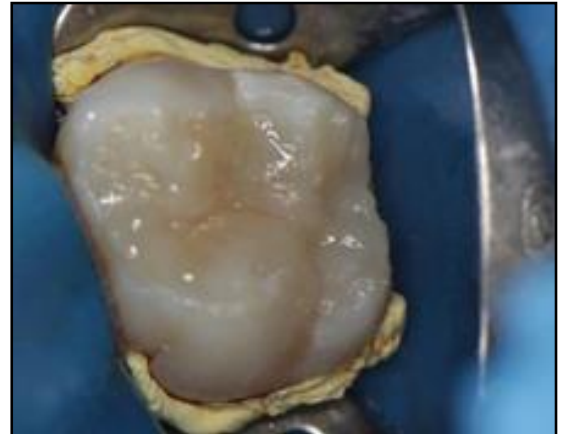

Fig 2j: Immediate post-operative photograph

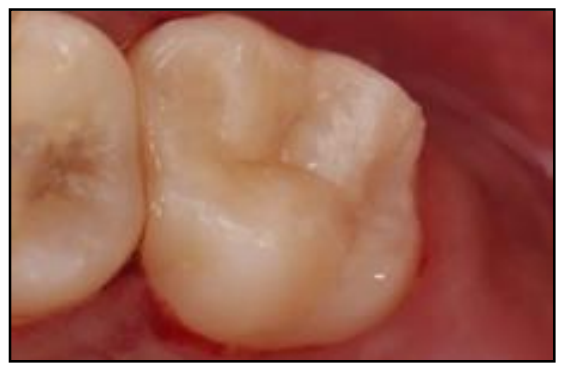

Fig 2k: After finishing and polishing

\section{Discussion}

With the advent of minimal intervention dentistry and greater impact on the aesthetic outlook there have been several modifications in terms of techniques as well as restorative procedures used in restoration of Class I cavities. There have been several factors which plays a pivotal role in the success of an ideal composite restoration, out of which two most important factors are polymerization shrinkage and proper cusp fossa relationship with the opposing arch.5 An ideal occlusion contour is desirable so as to produce ideal replica in fissures, marginal ridges and grooves such that it mimics the natural tooth.

The use of stamp technique in Class I carious lesion involves the replication of occlusal anatomy such that it should mimic the natural tooth. This is primarily indicated in teeth with intact cuspal planes and where carious extent is restricted to enamel and dentine. The major advantages of using this technique are less procedural time and instantly desired cuspfossa relationship is attainable6, less material consumption and easy to perform in finishing and polishing procedure and no need of additional instruments. It also increases the longevity of the restorations by its polymerization inhibiting effects ${ }^{[7]}$.

In this case flowable resin composite was used to record the occlusal details of the affected tooth. Besides this several other materials such as pit and fissure sealants, pattern resins, bite registration pastes can also be used. The main aim of this stamp technique is to record the minute details of the cavity and reproduce near -to -same occlusal outline.

No separating medium was used onto the occlusal surface of the tooth prior to the stamp preparation since it's a shallow lesion. However in moderate to deep lesions separating medium like Vaseline should be applied so as to cause easy removal of the stamp index and prevent any undue damage.

Even though this technique has several advantages, certain disadvantages of this technique are it requires skill and clinical astuteness so as to perform this procedure with utmost accuracy. Although this technique has been used in Class II cavities 1,8 , but majority of the cases where pits and fissures are preserved is Class I cavities5,9. Index is of less clinical efficacy for repeated restoration failure cases, it doesn't recreate imperfections such as deep pits and fissures since mimicking it in occlusal stamp is not possible. This technique is expensive because of microbrush and flowable composites. Incremental layering of composite placement is avoided to prevent polymerization shrinkage.

The prime importance of this procedure is to place the occlusal stamp correctly and with adequate precision with the main objective of obtaining accurate cusp -fossa relationship.10 Failure to perform this step will ultimately results in distortion of the restoration and ultimately nullifying the major objective of this technique.

\section{Conclusion}

Stamp technique is a favorable, operator friendly, given that operator is skillful, and biomimetic procedure for direct composite restorations. The precision of replicating the topography is much better than manual method and can be adapted to unconventional cavities as well.

\section{References}

1. Pompeu JGF, et al. Occlusal Stamp Technique for direct resin composite restoration: A Clinical Case Report". International Journal of Recent Scientific Research 2016;7:12427-12430.

2. Geena MG, Kevin RG. Building Proximal Contacts and Contours in Resin Composite Restorations: A Technical Report. Journal of Scientific Dentistry 2014;4(1):62-9.

3. Alshehadat SA, Halim MS, Carmen K, Fung CS. The stamp technique for direct Class II composite restorations: A case series. J Conserv Dent 2016;19:4903.

4. Ramseyer ST, Helbling C, Lussi A. Posterior vertical bite reconstructions of erosively worn dentitions and the "stamp technique" - A case series with a mean observation time of 40 months. J Adhes Dent 2015;17:283-9.

5. Martos J, Silveira LM, Ferrer-Luque CM, GonzálezLópez S. Restoration of posterior teeth using occlusal matrix technique. Indian J. Dent. Res 2010;21:596-599.

6. Leibenberg WH. Occlusal index-assisted restitution of aesthetic and functional anatomy in direct tooth- colored restorations. Quintessence International 1996;27:81-88.

7. Tambake NJ, et al. Stamp Technique- New perspective of Aesthetic Dentistry: A Case Report". IOSRJ 2017;16:49-51. 7.

8. Hamilton JC, et al. Evaluation of custom oFcclusal matrix technique for posterior light-cured composites". Operative Dentistry 1998;23:303-307.

9. Dawson EP. Evaluation, Diagnosis and treatment of Occlusal problems, 2 nd Ed., C.V. Mosby Co. (Toronto) 1989. 3.

10. Gross MD, Mathews JD. Occlusion in Restorative Dentistry, Technique and Theory, I st Ed. Churchill Livingston (NY): Longman Group limited 1982. 